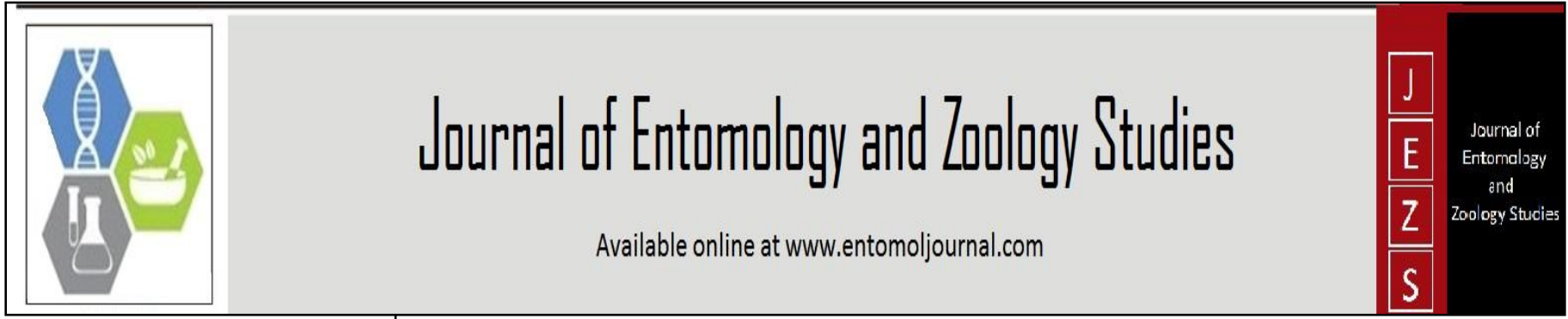

E-ISSN: 2320-7078

P-ISSN: 2349-6800

www.entomoljournal.com

JEZS 2020; 8(5): 1292-1300

(C) $2020 \mathrm{JEZS}$

Received: 21-07-2020

Accepted: 28-08-2020

SY Wankhede

Ph.D., Scholar, Department of

A gricultural Meteorology, College of

Agriculture, Pune, Mahatma Phule

Krishi Vidyapeeth, Rahuri,

Maharashtra, India

\section{SB Kharbade}

Associate Dean, College of

Agriculture, Nandurbar, Mahatm

Phule Krishi Vidyapeeth, Rahuri,

Maharashtra, India

VA Sthool

Associate Professor of

Hydrometeorology, Department of

Agricultural Meteorology, College of

Agriculture, Pune, Mahatma Phule

Krishi Vidyapeeth, Rahuri,

Maharashtra, India

\section{AA Shaikh}

Associate Professor of Agronomy,

Oilseed Research Station, Jalgaon.

Mahatma Phule Krishi

Vidyapeeth, Rahuri, Maharashtra,

India

\section{JD Jadhav}

Head, Department of Agricultural Meteorology, College of Agriculture, Pune, Mahatma Phule Krishi Vidyapeeth, Rahuri, Maharashtra India

\section{SN Hasabnis}

Associate Professor, Department of Plant Pathology, Division of Plant Pathology, College of Agriculture, Pune, Mahatma Phule Krishi Vidyapeeth, Rahuri, Maharashtra India

Rajkumar Bajolage

Scientist, Agricultural Entomology, ICAR- National Institute for Abiotic Stress Management, Baramati, Pune, Maharashtra, India
Corresponding Author:

SY Wankhede

Ph.D., Scholar, Department of Agricultural Meteorology, College of Agriculture, Pune, Mahatma Phule Krishi Vidyapeeth, Rahuri,

Maharashtra, India

\section{Population dynamics and forewarning models for prediction of population of tobacco leaf eating caterpillar, Spodoptera litura (Fab.) Under different sowing window and groundnut varieties}

\author{
SY Wankhede, SB Kharbade, VA Sthool, AA Shaikh, JD Jadhav, SN \\ Hasabnis, and Rajkumar Bajolage
}

DOI: https://doi.org/10.22271/j.ento.2020.v8.i5r.7687

Abstract

The correlation between weather parameter and population of tobacco leaf eating caterpillar, Spodoptera litura (Fab.) larva $\left(\mathrm{mrl}^{-1}\right)$ on different groundnut varieties at different sowing windows and forewarning models for prediction of population of tobacco leaf eating caterpillar, Spodoptera litura (Fab.) during kharif season, 2017 and 2018. The experiment was laid out in split plot design with three replications. The treatment comprised of four varieties viz., $\mathrm{V}_{1}$ : JL-501, $\mathrm{V}_{2}$ : RHRG-6083 (Phule Unnati), $\mathrm{V}_{3}$ : TAG-24 and $\mathrm{V}_{4}$ : JL-776 (Phule Bharati) as main plot and four sowing windows viz.. $\mathrm{S}_{1}: 25^{\text {rd }} \mathrm{MW}\left(18^{\text {th }}\right.$ to $24^{\text {th }}$ June), $\mathrm{S}_{2}: 26^{\text {th }} \mathrm{MW}\left(25^{\text {th }}\right.$ June to $\left.01^{\text {st }} J u l y\right), \mathrm{S}_{3}: 27^{\text {th }} \mathrm{MW}\left(2^{\text {nd }}\right.$ to $\left.8^{\text {th }} \mathrm{July}\right)$ and $\mathrm{S}_{4}: 28^{\text {th }} \mathrm{MW}\left(09^{\text {th }}\right.$ to $15^{\text {th }}$ July) as sub plot treatments. The correlation of weather parameters with incidence of Spodoptera litura showed that the population of Spodoptera litura was found to have significant and positive correlation with evening relative humidity whereas maximum temperature and rainfall showed negative correlation with seasonal incidence of Spodoptera litura. Prediction of Spodoptera litura populations in different sowing window based on regression equations $\left(\mathrm{R}^{2}\right) 72$ to 83 per cent validation based on different weather parameters for variety JL-501, $\left(\mathrm{R}^{2}\right) 66$ to 85 per cent validation based on different weather parameter for variety RHRG-6083, $\left(\mathrm{R}^{2}\right) 71$ to 87 per cent validation based on different weather parameter for variety TAG-24 and $\left(\mathrm{R}^{2}\right) 69$ to 84 per cent validation based on different weather parameter for variety JL-776 for the prediction of Spodoptera litura population.

Keywords: Correlation, forewarning models, groundnut, regression analysis, sowing window, Spodoptera litura (Fab.), variety

\section{Introduction}

Groundnut (Arachis hypogaea L.), it belongs to the family leguminacae and also known as peanut which ranks sixth among the oilseed crops and thirteenth among the food crops of the world. Groundnut occupies first place in India in respect of acreage and production. Insect pests of groundnut causes damage in both field and storage conditions. Due to the insect pests the annual loss estimated to be around Rs. 1500 million. About 115 insect pest species was reported in India, which causes damage to groundnut crop, in which only 9 species tobacco caterpillar, leaf miner, white grub, thrips, aphid, jassids,, gram caterpillar, red hairy caterpillar and termites are found to be economically important. The above-ground pests include tobacco caterpillar, Spodoptera litura Fab. and the groundnut leaf miner, Aproaerema modicella Deventer in Asia. Aphids and thrips transmit a number of viral diseases and more foliar damage caused by Jassids reported by Wightman and Amin (2008) ${ }^{[1]}$. Tobacco caterpillar, Spodoptera litura is one of the most important pest which is polyphagous and occur regularly in the field and horticultural crops reported by Murthy et al. (2006) ${ }^{[2]}$. At present it is major threats in intensive agriculture next to the gram caterpillar, Helicoverpa armigera and Spodoptera litura causes 26 to $100 \%$ yield loss under field conditions reported by Dhir et al. (1992) ${ }^{[3]}$; more than 180 crops reported by Isman et al. (2007) ${ }^{[4]}$. The groundnut bud borer, Anarsia ephippias (Meyerick) is minor pest of legumes and occur in Northern states of India. Terminal buds and shoots were preferred by larvae in which they make bore hole reported by Saheb et al. (2018) ${ }^{[5]}$. 
Most of the pesticides that are used in cotton, rice, pulses, oilseeds and vegetable crops had shown ill effects on human health and environment reported by Raghunathan (1996) ${ }^{[6]}$.

Different strategies have to be involved for keeping the pest in check and stabilizing the productivity of the cropping system. The sowing window is one of the crop habitat desertification that is to be looked into, to minimize the incidence of insect pests on groundnut crop so that its yield can be enhanced. The sowing window takes the advantage of the absence of the pest or avoids susceptible stage of the crop. It prevents carryover of pests from early sown crop to late sown crop and prevents buildup of damaging populations reported by Singh (1999) ${ }^{[7]}$. The pest forewarning model was able to predict the percent population of Spodoptera litura Fab. for different groundnut varieties and sowing windows with good $r^{2}$ values. Keeping these facts in view, the correlation between weather parameter and population of tobacco leaf eating caterpillar, Spodoptera litura (Fab.) larva $\left(\mathrm{mrl}^{-1}\right)$ on different groundnut varieties at different sowing windows and development of forewarning models for prediction of population of tobacco leaf eating caterpillar, Spodoptera litura (Fab.) was studied during kharif season, 2017 and 2018.

\section{Material and Methods \\ Location of the experimental site and climatic condition}

The experiment was conducted in a Split plot design with three replications and sixteen treatment combinations formed considering different varieties and sowing windows. The gross and net plot size was $4.5 \times 4.5 \mathrm{~m}^{2}$ and $3.6 \times 3.6 \mathrm{~m}^{2}$, respectively for two consecutive years at Department of Agricultural Meteorology farm, College of Agriculture, Pune during kharif, 2017 and 2018. The geographical location of the site (Pune) was $18^{\circ} 32^{\prime} \mathrm{N}$, latitude; $73^{\circ} 51 \mathrm{E}$, longitude and $557.7 \mathrm{~m}$ above mean sea level (MSL). The soil is medium black having depth of about $1 \mathrm{~m}$. The experimental site is situated in the sub-tropical region (Plain Zone) on the latitude $18^{\circ} 22^{\prime} \mathrm{N}$ and longitude $73^{\circ} 51^{\prime} \mathrm{E}$ and having an altitude of $557.7 \mathrm{~m}$ above the mean sea level. The average annual rainfall of Pune is $675 \mathrm{~mm}$, which is distributed from second fortnight of June to second fortnight of October. Out of total rainfall, about 75 per cent is received from June to September from south-west monsoon, while remaining is received from northeast monsoon during October and November. Urea and single superphosphate were used as source of $\mathrm{N}$ and $\mathrm{P}$ and applied as per recommended dose i.e. $25 \mathrm{~kg} \mathrm{~N}$ and $50 \mathrm{~kg} \mathrm{P}_{2} \mathrm{O}_{5}$. Seed of groundnut was inoculated with Rhizobium culture @ $250 \mathrm{~g} 10$ $\mathrm{kg}^{-1}$ seed.

\section{Number of Spodoptera litura larvae m row length ${ }^{-1}$ week $^{-1}$}

Number of Spodoptera litura larvae m row length ${ }^{-1}$ week $^{-1}$ observations on larval population was made at three randomly selected spots of one meter row length in each plot, leaving the border rows. Larval count was made by shaking plants gently over a white cloth placed between the rows.

The effect of weather factors viz; maximum and minimum temperatures $\left({ }^{0} \mathrm{C}\right)$, relative humidity in per cent (morning and evening), bright sunshine hours, rainfall ( $\mathrm{mm}$ ) and rainy days, wind speed, evaporation rate from meteorological weeks $25^{\text {th }}$ to $44^{\text {th }}$ in kharif; 2017 and 2018, on Spodoptera litura were studied. The influence of weather parameters on pest population and was estimated by using prediction equation as, $\mathrm{Y}=\mathrm{a}+\mathrm{b} 1 \times 1+\mathrm{b} 2 \times 2+\mathrm{b} 3 \times 3+\ldots \ldots .+\mathrm{bnxn}$.

Where, $\mathrm{Y}=$ Spodoptera litura population, ' $\mathrm{a}$ ' as constant and ' $b$ ' as regression coefficients of independent variable ' $x$ '.
Correlation analysis of mean weather parameters and mean data of two years on resultant crop growth characters, yield attributes and yield including water utilized for analysis. Snedecor and Cochron (1968) ${ }^{[8]}$ studied the influence of weather parameters on crop growth yield and yield attributes, initially simple correlations were carried out. After establishing the relationship between weather parameters and Spodoptera litura multiple regression analysis Drapper and Smith (1998) ${ }^{\text {[9] }}$ was carried out considering those weather parameters that had significant influence on Spodoptera litura of groundnut. The forewarning models for different sowing windows and varieties of groundnut were worked out by statistical analysis using SPSS8.0 software by multiple linear regression method. The correlation coefficient values were further used for results and discussion.

\section{Result and Discussion}

Population dynamics of Spodoptera litura on groundnut

The study was conducted during both the years of kharif season (2017 and 2018). During the course of study, the incidence of Spodoptera litura was recorded on different groundnut varieties were sown at different sowing windows. The incidence of Spodoptera litura was recorded all varieties during the year 2017 and 2018 in Table 1 to 4 across all the windows of sowing.

Population dynamics of Spodoptera litura larva $\left(\mathrm{mrl}^{-1}\right)$ on different groundnut varieties under sowing window $\left(S_{1}\right)$ $2^{\text {th }}$ MW

During the year 2017, first sowing window $25^{\text {th }} \mathrm{MW}\left(\mathrm{S}_{1}\right)$ with the varieties JL-501 $\left(\mathrm{V}_{1}\right)$, RHRG-6083 $\left(\mathrm{V}_{2}\right)$ and TAG-24 $\left(\mathrm{V}_{3}\right)$ and JL-776 $\left(\mathrm{V}_{4}\right)$ the mean incidence was 2.97, 1.93, 3.24 and 1.34 larvas $\left(\mathrm{mrl}^{-1}\right)$ and which were at peak with 4.51, 2.93, 4.92 and 2.03 larvas $\left(\mathrm{mrl}^{-1}\right)$. During the year 2018, the first sowing window $25^{\text {th }} \mathrm{MW}\left(\mathrm{S}_{1}\right)$ with the varieties $\mathrm{JL}-501\left(\mathrm{~V}_{1}\right)$, RHRG-6083 $\left(\mathrm{V}_{2}\right)$ and TAG-24 $\left(\mathrm{V}_{3}\right)$ and JL-776 $\left(\mathrm{V}_{4}\right)$ recorded the mean incidence of larva $\left(\mathrm{mrl}^{-1}\right)$ was $2.62,1.71,2.86$ and 1.18 , which was peak with $4.03,2.59,4.35$ and 1.80 larva $\left(\mathrm{mrl}^{-1}\right)$, resulting the peak population of larva $\left(\mathrm{mrl}^{-1}\right)$ was noticed at $34^{\text {th }} \mathrm{MW}$ with sowing window $25^{\text {th }} \mathrm{MW}$, during both the year 2017 and 2018. These results are in conformity with the results of Rathod (2006) ${ }^{[10]}$, Arvind kumar (2014) ${ }^{[11]}$, Harish et al. (2014) ${ }^{[12]}$, Roopa and Ashok kumar (2014) ${ }^{[13]}$ and Nath et al. (2017) ${ }^{[14]}$.

\section{Population dynamics of Spodoptera litura larva $\left(\mathrm{mrl}^{-1}\right)$ on different groundnut varieties under sowing window $\left(\mathbf{S}_{2}\right)$ 26 $^{\text {th }}$ MW}

During the year 2017, second sowing window $26^{\text {th }} \mathrm{MW}\left(\mathrm{S}_{2}\right)$ with the varieties JL-501 $\left(\mathrm{V}_{1}\right)$, RHRG-6083 $\left(\mathrm{V}_{2}\right)$ and TAG-24 $\left(\mathrm{V}_{3}\right)$ and JL-776 $\left(\mathrm{V}_{4}\right)$ the mean incidence was $2.13,1.38,2.32$, and 0.96 larvas $\left(\mathrm{mrl}^{-1}\right)$ and which were at peak with 3.57 , 2.32, 3.89 and 1.61 larvas $\left(\mathrm{mrl}^{-1}\right)$. During the year 2018, the second sowing window $26^{\text {th }} \mathrm{MW}\left(\mathrm{S}_{2}\right)$ with the varieties $\mathrm{JL}$ $501\left(\mathrm{~V}_{1}\right)$, RHRG-6083 $\left(\mathrm{V}_{2}\right)$ and TAG-24 $\left(\mathrm{V}_{3}\right)$ and JL-776 $\left(\mathrm{V}_{4}\right)$ recorded the mean incidence of larva $\left(\mathrm{mrl}^{-1}\right)$ was 1.90 , $1.22,2.05$ and 0.85 , which was peak with 3.19, 2.05, 3.44 and 1.42 larva $\left(\mathrm{mrl}^{-1}\right)$, resulting the peak population of larva $\left(\mathrm{mrl}^{-}\right.$ ${ }^{1}$ ) was noticed at $35^{\text {th }} \mathrm{MW}$ with sowing window $26^{\text {th }} \mathrm{MW}$, during both the year 2017 and 2018. These results are in conformity with the results of Rathod (2006) ${ }^{[10]}$, Arvind kumar (2014) ${ }^{[11]}$, Harish et al. (2014) ${ }^{[12]}$, Roopa and Ashok kumar (2014) ${ }^{[13]}$ and Nath et al. (2017) ${ }^{[14]}$. 
Population dynamics of Spodoptera litura larva $\left(\mathrm{mrl}^{-1}\right)$ on different groundnut varieties under sowing window $\left(\mathbf{S}_{3}\right)$ $27^{\text {th }}$ MW

During the year 2017, third sowing window $27^{\text {th }} \mathrm{MW}\left(\mathrm{S}_{3}\right)$ with the varieties JL-501 $\left(\mathrm{V}_{1}\right)$, RHRG-6083 $\left(\mathrm{V}_{2}\right)$ and TAG-24 $\left(\mathrm{V}_{3}\right)$ and JL-776 $\left(\mathrm{V}_{4}\right)$ the mean incidence was 2.43, 1.58, 2.65, and 1.10 larvas $\left(\mathrm{mrl}^{-1}\right)$ and which were at peak with 4.34 , 2.82, 4.73 and 1.96 larvas $\left(\mathrm{mrl}^{-1}\right)$. During the year 2018, the third sowing window $27^{\text {th }} \mathrm{MW}\left(\mathrm{S}_{3}\right)$ with the varieties JL-501 $\left(\mathrm{V}_{1}\right)$, RHRG-6083 $\left(\mathrm{V}_{2}\right)$ and TAG-24 $\left(\mathrm{V}_{3}\right)$ and JL-776 $\left(\mathrm{V}_{4}\right)$ recorded the mean incidence of larva $\left(\mathrm{mrl}^{-1}\right)$ was $2.17,1.40$, 2.35 and 0.97 which was peak with $3.88,2.49,4.19$ and 1.73 larva $\left(\mathrm{mrl}^{-1}\right)$, resulting the peak population of larva $\left(\mathrm{mrl}^{-1}\right)$ was noticed at $36^{\text {th }} \mathrm{MW}$ with sowing window $27^{\text {th }} \mathrm{MW}$, during both the year 2017 and 2018. These results are in conformity with the results of Rathod (2006) ${ }^{[10]}$, Arvind Kumar (2014) ${ }^{[11]}$, Harish et al. (2014) ${ }^{[12]}$, Roopa and Ashok Kumar (2014) ${ }^{[13]}$ and Nath et al. (2017) ${ }^{[14]}$.
Population dynamics of Spodoptera litura larva $\left(\mathrm{mrl}^{-1}\right)$ on different groundnut varieties under sowing window $\left(\mathbf{S}_{4}\right)$ $28^{\text {th }}$ MW

During the year 2017, fourth sowing window $28^{\text {th }} \mathrm{MW}\left(\mathrm{S}_{4}\right)$ with the varieties JL-501 $\left(\mathrm{V}_{1}\right)$, RHRG-6083 $\left(\mathrm{V}_{2}\right)$ and TAG-24 $\left(\mathrm{V}_{3}\right)$ and JL-776 $\left(\mathrm{V}_{4}\right)$ the mean incidence was 3.36, 2.18, 3.66, and 1.51 larvas $\left(\mathrm{mrl}^{-1}\right)$ and which were at peak with 5.23, 3.39, 5.70 and 2.36 larvas $\left(\mathrm{mrl}^{-1}\right)$. During the year 2018, the fourth sowing window $28^{\text {th }} \mathrm{MW}\left(\mathrm{S}_{4}\right)$ with the varieties JL-501 $\left(\mathrm{V}_{1}\right)$, RHRG-6083 $\left(\mathrm{V}_{2}\right)$ and TAG-24 $\left(\mathrm{V}_{3}\right)$ and JL-776 $\left(\mathrm{V}_{4}\right)$ recorded the mean incidence of larva $\left(\mathrm{mrl}^{-1}\right)$ was 3.00, 1.93, 3.24 and 1.34 which was peak with 4.67, 3.00, 5.04 and 2.09 larva $\left(\mathrm{mrl}^{-1}\right)$, resulting the peak population of larva $\left(\mathrm{mrl}^{-1}\right)$ was noticed at $36^{\text {th }} \mathrm{MW}$ with sowing window $28^{\text {th }} \mathrm{MW}$, during both the year 2017 and 2018. These results are in conformity with the results of Rathod (2006) ${ }^{[10]}$, Arvind Kumar (2014) ${ }^{[11]}$, Harish et al. (2014) ${ }^{[12]}$, Roopa and Ashok Kumar (2014) ${ }^{[13]}$ and Nath et al. (2017) ${ }^{[14]}$.

Table 1: Mean number of larva per meter row length $\left(\mathrm{mrl}^{-1)}\right.$ of Spodoptera litura on groundnut variety JL-501 as influenced by different sowing windows

\begin{tabular}{|c|c|c|c|c|c|c|c|c|c|}
\hline \multirow{3}{*}{ Sr. No. } & \multirow{3}{*}{ MW } & \multicolumn{8}{|c|}{ Number of larva per meter row length $\left(\mathrm{mrl}^{-1}\right)$} \\
\hline & & \multicolumn{4}{|c|}{2017} & \multicolumn{4}{|c|}{2018} \\
\hline & & $\mathrm{S}_{1}\left(25^{\text {th }} \mathrm{MW}\right)$ & $\mathbf{S}_{2}\left(\mathbf{2 6}^{\text {th }} \mathrm{MW}\right)$ & $\mathbf{S}_{3}\left(27^{\text {th }} M W\right)$ & $\mathrm{S}_{4}\left(28^{\text {th }} \mathrm{MW}\right)$ & $\mathbf{S}_{1}\left(2^{\text {th }} \mathrm{MW}\right)$ & $\mathbf{S}_{2}\left(\mathbf{2 6}^{\text {th }} \mathrm{MW}\right)$ & $\mathrm{S}_{3}\left(2^{\text {th }} \mathrm{MW}\right)$ & $\mathrm{S}_{4}\left(28^{\text {th }} \mathrm{MW}\right)$ \\
\hline 1 & 29 & 0.00 & - & - & - & 0.00 & - & - & - \\
\hline 2 & 30 & 1.47 & 0.00 & - & - & 1.31 & 0.00 & - & - \\
\hline 3 & 31 & 2.27 & 0.58 & 0.00 & - & 2.03 & 0.52 & 0.00 & - \\
\hline 4 & 32 & 3.24 & 1.10 & 1.00 & 0.87 & 2.89 & 0.98 & 0.89 & 0.78 \\
\hline 5 & 33 & 4.41 & 2.05 & 1.39 & 2.22 & 3.94 & 1.83 & 1.24 & 1.98 \\
\hline 6 & 34 & 4.51 & 3.07 & 2.22 & 2.76 & 4.03 & 2.74 & 1.98 & 2.46 \\
\hline 7 & 35 & 4.20 & 3.57 & 3.48 & 3.59 & 3.75 & 3.19 & 3.11 & 3.21 \\
\hline 8 & 36 & 3.66 & 3.37 & 4.34 & 5.23 & 3.27 & 3.01 & 3.88 & 4.67 \\
\hline 9 & 37 & 3.26 & 2.90 & 3.80 & 4.75 & 2.91 & 2.59 & 3.39 & 4.24 \\
\hline 10 & 38 & 2.68 & 2.52 & 3.24 & 4.11 & 2.39 & 2.25 & 2.89 & 3.67 \\
\hline 11 & 39 & - & 1.95 & 2.74 & 3.61 & - & 1.74 & 2.45 & 3.22 \\
\hline 12 & 40 & - & - & 2.25 & 2.81 & - & - & 2.01 & 2.51 \\
\hline 13 & 41 & - & - & - & 1.33 & - & - & - & 1.19 \\
\hline 14 & 42 & - & - & - & - & - & - & - & - \\
\hline 15 & 43 & - & - & - & - & - & - & - & - \\
\hline 16 & 44 & - & - & - & - & - & - & - & - \\
\hline \multicolumn{2}{|c|}{ Mean } & 2.97 & 2.13 & 2.43 & 3.36 & 2.65 & 1.90 & 2.17 & 3.00 \\
\hline
\end{tabular}

Table 2: Mean number of larva per meter row length $\left(\mathrm{mrl}^{-1)}\right.$ of Spodoptera litura on groundnut variety RHRG-6083 as influenced by different sowing windows

\begin{tabular}{|c|c|c|c|c|c|c|c|c|c|}
\hline \multirow{3}{*}{ Sr. No. } & \multirow{3}{*}{ MW } & \multicolumn{8}{|c|}{ Number of larva per meter row length $\left(\mathrm{mrl}^{-1}\right)$} \\
\hline & & \multicolumn{4}{|c|}{2017} & \multicolumn{4}{|c|}{2018} \\
\hline & & $\mathrm{S}_{1}\left(25^{\text {th }} \mathrm{MW}\right)$ & $\mathbf{S}_{2}\left(\mathbf{2 6}^{\text {th }} \mathrm{MW}\right)$ & $S_{3}\left(27^{\text {th }} M W\right)$ & $\mathrm{S}_{4}\left(28^{\text {th }} \mathrm{MW}\right)$ & $\mathrm{S}_{1}\left(25^{\text {th }} \mathrm{MW}\right)$ & $\mathbf{S}_{2}\left(2^{\text {th }} \mathrm{MW}\right)$ & $\mathrm{S}_{3}\left(27^{\text {th }} \mathrm{MW}\right)$ & $\mathrm{S}_{4}\left(28^{\text {th }} \mathrm{MW}\right)$ \\
\hline 1 & 29 & 0.00 & - & - & - & 0.00 & - & - & - \\
\hline 2 & 30 & 0.95 & 0.00 & - & - & 0.84 & 0.00 & - & - \\
\hline 3 & 31 & 1.47 & 0.38 & 0.00 & - & 1.30 & 0.33 & 0.00 & - \\
\hline 4 & 32 & 2.10 & 0.71 & 0.65 & 0.56 & 1.86 & 0.63 & 0.57 & 0.50 \\
\hline 5 & 33 & 2.86 & 1.33 & 0.90 & 1.44 & 2.53 & 1.18 & 0.80 & 1.27 \\
\hline 6 & 34 & 2.93 & 1.99 & 1.44 & 1.79 & 2.59 & 1.76 & 1.27 & 1.58 \\
\hline 7 & 35 & 2.73 & 2.32 & 2.26 & 2.33 & 2.41 & 2.05 & 2.00 & 2.06 \\
\hline 8 & 36 & 2.37 & 2.19 & 2.82 & 3.39 & 2.10 & 1.93 & 2.49 & 3.00 \\
\hline 9 & 37 & 2.12 & 1.88 & 2.47 & 3.08 & 1.87 & 1.67 & 2.18 & 2.73 \\
\hline 10 & 38 & 1.74 & 1.64 & 2.10 & 2.67 & 1.54 & 1.45 & 1.86 & 2.36 \\
\hline 11 & 39 & - & 1.27 & 1.78 & 2.34 & - & 1.12 & 1.57 & 2.07 \\
\hline 12 & 40 & - & - & 1.46 & 1.82 & - & - & 1.29 & 1.61 \\
\hline 13 & 41 & - & - & - & 0.86 & - & - & - & 0.76 \\
\hline 14 & 42 & - & - & - & - & - & - & - & - \\
\hline 15 & 43 & - & - & - & - & - & - & - & - \\
\hline 16 & 44 & - & - & - & - & - & - & - & - \\
\hline \multicolumn{2}{|c|}{ Mean } & 1.93 & 1.38 & 1.58 & 2.18 & 1.71 & 1.22 & 1.40 & 1.93 \\
\hline
\end{tabular}


Table 3: Mean number of larva per meter row length $\left(\mathrm{mrl}^{-1)}\right.$ of Spodoptera litura on groundnut variety TAG-24 as influenced by different sowing windows

\begin{tabular}{|c|c|c|c|c|c|c|c|c|c|}
\hline \multirow{3}{*}{ Sr. No. } & \multirow{3}{*}{ MW } & \multicolumn{8}{|c|}{ Number of larva per meter row length $\left(\mathrm{mrl}^{-1}\right)$} \\
\hline & & \multicolumn{4}{|c|}{2017} & \multicolumn{4}{|c|}{2018} \\
\hline & & $S_{1}\left(25^{\text {th }} M W\right)$ & $\mathbf{S}_{2}\left(2^{\text {th }} \mathrm{MW}\right)$ & $\mathrm{S}_{3}\left(27^{\text {th }} \mathrm{MW}\right)$ & $S_{4}\left(28^{\text {th }} M W\right)$ & $\mathrm{S}_{1}\left(25^{\text {th }} \mathrm{MW}\right)$ & $S_{2}\left(26^{\text {th }} M W\right)$ & $\mathrm{S}_{3}\left(27^{\text {th }} \mathrm{MW}\right)$ & $S_{4}\left(28^{\text {th }} M W\right)$ \\
\hline 1 & 29 & 0.00 & - & - & - & $\begin{array}{l}0.00 \\
\end{array}$ & - & - & - \\
\hline 2 & 30 & 1.60 & 0.00 & - & - & 1.42 & 0.00 & - & - \\
\hline 3 & 31 & 2.47 & 0.63 & 0.00 & - & 2.19 & 0.56 & 0.00 & - \\
\hline 4 & 32 & 3.53 & 1.20 & 1.09 & 0.95 & 3.13 & 1.06 & 0.96 & 0.84 \\
\hline 5 & 33 & 4.81 & 2.23 & 1.52 & 2.42 & 4.25 & 1.98 & 1.34 & 2.14 \\
\hline 6 & 34 & 4.92 & 3.35 & 2.42 & 3.01 & 4.35 & 2.96 & 2.14 & 2.66 \\
\hline 7 & 35 & 4.58 & 3.89 & 3.79 & 3.91 & 4.05 & 3.44 & 3.36 & 3.46 \\
\hline 8 & 36 & 3.99 & 3.67 & 4.73 & 5.70 & 3.53 & 3.25 & 4.19 & 5.04 \\
\hline 9 & 37 & 3.55 & 3.16 & 4.14 & 5.18 & 3.14 & 2.80 & 3.67 & 4.58 \\
\hline 10 & 38 & 2.92 & 2.75 & 3.53 & 4.48 & 2.59 & 2.43 & 3.13 & 3.96 \\
\hline 11 & 39 & - & 2.13 & 2.99 & 3.93 & - & 1.88 & 2.64 & 3.48 \\
\hline 12 & 40 & - & - & 2.45 & 3.06 & - & - & 2.17 & 2.71 \\
\hline 13 & 41 & - & - & - & 1.45 & - & - & - & 1.28 \\
\hline 14 & 42 & - & - & - & - & - & - & - & - \\
\hline 15 & 43 & - & - & - & - & - & - & - & - \\
\hline 16 & 44 & - & - & - & - & - & - & - & - \\
\hline \multicolumn{2}{|c|}{ Mean } & 3.24 & 2.32 & 2.65 & 3.66 & 2.86 & 2.05 & 2.35 & 3.24 \\
\hline
\end{tabular}

Table 4: Mean number of larva per meter row length $\left(\mathrm{mrl}^{-1)}\right.$ of Spodoptera litura on groundnut variety JL-776 as influenced by different sowing windows

\begin{tabular}{|c|c|c|c|c|c|c|c|c|c|}
\hline \multirow{3}{*}{ Sr. No. } & \multirow{3}{*}{ MW } & \multicolumn{8}{|c|}{ Number of larva per meter row length $\left(\mathrm{mrl}^{-1}\right)$} \\
\hline & & \multicolumn{4}{|c|}{2017} & \multicolumn{4}{|c|}{2018} \\
\hline & & $\mathrm{S}_{1}\left(25^{\text {th }} \mathrm{MW}\right)$ & $\mathbf{S}_{2}\left(\mathbf{2 6}^{\text {th }} \mathrm{MW}\right)$ & $\mathbf{S}_{3}\left(27^{\text {th }} \mathrm{MW}\right)$ & $\mathrm{S}_{4}\left(28^{\text {th }} \mathrm{MW}\right)$ & $\mathrm{S}_{1}\left(25^{\text {th }} \mathrm{MW}\right)$ & $S_{2}\left(26^{\text {th }} M W\right)$ & $\mathrm{S}_{3}\left(27^{\text {th }} \mathrm{MW}\right)$ & $\mathrm{S}_{4}\left(2^{\text {th }} \mathrm{MW}\right)$ \\
\hline 1 & 29 & 0.00 & & & & 0.00 & - & - & - \\
\hline 2 & 30 & 0.66 & 0.00 & & & 0.59 & 0.00 & - & - \\
\hline 3 & 31 & 1.02 & 0.26 & 0.00 & & 0.91 & 0.23 & 0.00 & - \\
\hline 4 & 32 & 1.46 & 0.50 & 0.45 & 0.39 & 1.29 & 0.44 & 0.40 & 0.35 \\
\hline 5 & 33 & 1.99 & 0.92 & 0.63 & 1.00 & 1.76 & 0.82 & 0.55 & 0.89 \\
\hline 6 & 34 & 2.03 & 1.38 & 1.00 & 1.24 & 1.80 & 1.22 & 0.89 & 1.10 \\
\hline 7 & 35 & 1.89 & 1.61 & 1.57 & 1.62 & 1.67 & 1.42 & 1.39 & 1.43 \\
\hline 8 & 36 & 1.65 & 1.52 & 1.96 & 2.36 & 1.46 & 1.34 & 1.73 & 2.09 \\
\hline 9 & 37 & 1.47 & 1.31 & 1.71 & 2.14 & 1.30 & 1.16 & 1.52 & 1.89 \\
\hline 10 & 38 & 1.21 & 1.14 & 1.46 & 1.85 & 1.07 & 1.00 & 1.29 & 1.64 \\
\hline 11 & 39 & - & 0.88 & 1.23 & 1.63 & - & 0.78 & 1.09 & 1.44 \\
\hline 12 & 40 & - & - & 1.01 & 1.27 & - & - & 0.90 & 1.12 \\
\hline 13 & 41 & - & - & - & 0.60 & - & - & - & 0.53 \\
\hline 14 & 42 & - & - & - & - & - & - & - & - \\
\hline 15 & 43 & - & - & - & - & - & - & - & - \\
\hline 16 & 44 & - & - & - & - & - & - & - & - \\
\hline \multicolumn{2}{|c|}{ Mean } & 1.34 & 0.96 & 1.10 & 1.51 & 1.18 & 0.85 & 0.97 & 1.34 \\
\hline
\end{tabular}

\section{Number of adults}

The moth activity was monitored using sex pheromone traps for different treatments from $25^{\text {th }} \mathrm{MW}$ to $41^{\text {st }} \mathrm{MW}$ during 2017 and 2018 in groundnut and data are given in Table. 5. Only two pheromone traps was installed in the field during the experimental period in both the years. Data pertaining to adult catches in pheromone trap was not analyzed statistically.
Inferences are based on mean values. The pheromone trap catches of Spodoptera litura was the highest during 70 DAS (5 moths trap ${ }^{-1}$ and 4.5 moths trap ${ }^{-1}$ ) in 2017 and 2018. The moth activity increased from 14 DAS to 70 DAS and thereafter decreased up to harvest. The study revealed that there was a wide fluctuation in the pattern of moth catches across the weeks.

Table 5: Number of adults in pheromone trap of soybean as influenced by different treatments

\begin{tabular}{|c|c|c|c|}
\hline \multicolumn{5}{|c|}{ Number of adults of Spodoptera litura } \\
\hline MW & $\mathbf{2 0 1 7}$ & $\mathbf{2 0 1 8}$ & MEAN \\
\hline 25 & 0.00 & 0.00 & 0.00 \\
\hline 26 & 0.00 & 0.00 & 0.00 \\
\hline 27 & 0.00 & 0.00 & 0.00 \\
\hline 28 & 0.00 & 0.00 & 0.00 \\
\hline 29 & 0.00 & 0.00 & 0.00 \\
\hline 30 & 0.00 & 0.00 & 0.00 \\
\hline 31 & 0.00 & 0.00 & 0.00 \\
\hline 32 & 0.50 & 0.00 & 0.25 \\
\hline 33 & 1.50 & 1.00 & 1.25 \\
\hline
\end{tabular}




\begin{tabular}{|c|c|c|c|}
\hline 34 & 2.20 & 1.50 & 1.85 \\
\hline 35 & 3.50 & 2.50 & 3.00 \\
\hline 36 & 4.00 & 3.00 & 3.50 \\
\hline 37 & 5.00 & 4.50 & 4.75 \\
\hline 38 & 4.00 & 4.00 & 4.00 \\
\hline 39 & 2.50 & 2.00 & 2.25 \\
\hline 40 & 1.50 & 1.00 & 1.25 \\
\hline 41 & 1.00 & 0.50 & 0.75 \\
\hline
\end{tabular}

Correlation between weather parameter and population of Spodoptera litura larva $\left(\mathrm{mrl}^{-1}\right)$ on different groundnut varieties at different sowing windows and forewarning models for prediction of population of Spodoptera litura

The influence of different weather parameters viz., maximum and minimum temperature, morning and evening relative humidity, wind speed evaporation and rainfall on the seasonal population of Spodoptera litura larva $\left(\mathrm{mrl}^{-1}\right)$ was observed by working out correlation coefficient (r) (Table 6 and 7) and forewarning models were developed are given in Table 8.

Results of the cumulative correlation showed that the population of thrips was found to have significant and positive correlation with minimum temperature, morning and evening relative humidity, wind speed and bright sunshine hours whereas maximum and minimum temperature, evaporation and rainfall showed negative correlation. On the basis of different sowing windows and varieties, the incidence of Spodoptera litura with weather factors was studies.

Table 6: Correlation between different weather parameters and population of Spodoptera litura larva per meter row length $\left(\mathrm{mrl}^{-1}\right)$ during kharif $^{-}$ 2017

\begin{tabular}{|c|c|c|c|c|c|c|c|c|c|}
\hline \multicolumn{2}{|c|}{ Treatment } & \multicolumn{8}{|c|}{ r'values } \\
\hline Sowing window & Variety & Tmax & Tmin & RH-I & RH-II & RAIN & WS & Epan & B S S \\
\hline $\mathrm{S}_{1}-25^{\text {th }} \mathrm{MW}$ & \multirow{4}{*}{$\mathrm{V}_{1-J L-501}$} & $-0.546^{*}$ & 0.216 & -0.356 & 0.287 & -0.202 & 0.458 & 0.093 & -0.314 \\
\hline $\mathrm{S}_{2}-26^{\text {th }} \mathrm{MW}$ & & -0.484 & 0.088 & -0.122 & 0.255 & -0.034 & 0.205 & 0.230 & -0.130 \\
\hline $\mathrm{S}_{3}-27^{\text {th }} \mathrm{MW}$ & & -0.279 & 0.047 & 0.038 & 0.232 & 0.019 & -0.011 & 0.345 & 0.055 \\
\hline $\mathrm{S}_{4}-28^{\text {th }} \mathrm{MW}$ & & -0.185 & 0.087 & 0.002 & 0.175 & -0.059 & -0.069 & 0.360 & 0.040 \\
\hline $\mathrm{S}_{1}-25^{\text {th }} \mathrm{MW}$ & \multirow{4}{*}{$V_{2}-$ RHRG-6083 } & $-0.554^{*}$ & 0.214 & -0.365 & 0.290 & -0.203 & 0.467 & 0.093 & -0.310 \\
\hline $\mathrm{S}_{2}-26^{\text {th }} \mathrm{MW}$ & & -0.481 & 0.088 & -0.123 & 0.252 & -0.036 & 0.205 & 0.228 & -0.132 \\
\hline $\mathrm{S}_{3}-27^{\text {th }} \mathrm{MW}$ & & -0.288 & 0.048 & 0.036 & 0.234 & 0.021 & -0.005 & 0.341 & 0.049 \\
\hline $\mathrm{S}_{4}-28^{\text {th }} \mathrm{MW}$ & & -0.187 & 0.087 & 0.003 & 0.177 & -0.058 & -0.068 & 0.361 & 0.040 \\
\hline $\mathrm{S}_{1}-25^{\text {th }} \mathrm{MW}$ & \multirow{4}{*}{$\mathrm{V}_{3}-\mathrm{TAG}-24$} & $-0.550^{*}$ & 0.216 & -0.362 & 0.289 & -0.203 & 0.464 & 0.092 & -0.313 \\
\hline $\mathrm{S}_{2}-26^{\text {th }} \mathrm{MW}$ & & -0.486 & 0.088 & -0.120 & 0.257 & -0.033 & 0.206 & 0.231 & -0.128 \\
\hline $\mathrm{S}_{3}-27^{\text {th }} \mathrm{MW}$ & & -0.289 & 0.048 & 0.035 & 0.234 & 0.022 & -0.004 & 0.341 & 0.048 \\
\hline $\mathrm{S}_{4}-28^{\text {th }} \mathrm{MW}$ & & -0.186 & 0.087 & 0.002 & 0.175 & -0.059 & -0.069 & 0.360 & 0.040 \\
\hline $\mathrm{S}_{1}-25^{\text {th }} \mathrm{MW}$ & \multirow{4}{*}{ V4-JL-776 } & $-0.545^{*}$ & 0.220 & -0.358 & 0.288 & -0.204 & 0.459 & 0.092 & -0.316 \\
\hline $\mathrm{S}_{2}-26^{\text {th }} \mathrm{MW}$ & & -0.470 & 0.088 & -0.130 & 0.241 & -0.044 & 0.205 & 0.222 & -0.142 \\
\hline $\mathrm{S}_{3}-27^{\text {th }} \mathrm{MW}$ & & -0.280 & 0.047 & 0.038 & 0.232 & 0.019 & -0.011 & 0.344 & 0.055 \\
\hline $\mathrm{S}_{4-} 28^{\text {th }} \mathrm{MW}$ & & -0.159 & 0.092 & -0.006 & 0.157 & -0.074 & -0.086 & 0.351 & 0.030 \\
\hline
\end{tabular}

Tmax- Maximum temperature, Tmin- Minimum temperatur, RH-I- Morning humidity, RH-II- Evening humidity, WS- Wind speed, RFRainfall, Epan- Evaporation, BSS- Bright sunshine hours

Table 7: Correlation between different weather parameters and population of Spodoptera litura larva per meter row length $\left(\mathrm{mrl}^{-1}\right)$ during kharif $^{-}$ 2018

\begin{tabular}{|c|c|c|c|c|c|c|c|c|c|}
\hline \multicolumn{2}{|c|}{ Treatment } & \multicolumn{8}{|c|}{ r'values } \\
\hline Sowing window & Variety & Tmax & Tmin & RH-I & RH-II & RAIN & WS & Epan & B S S \\
\hline $\mathrm{S}_{1}-25^{\text {th }} \mathrm{MW}$ & \multirow{4}{*}{$\mathrm{V}_{1}-\mathrm{JL}-501$} & $-0.731 *$ & 0.291 & 0.090 & $0.703 *$ & 0.066 & $0.598^{*}$ & $-0.540 *$ & -0.483 \\
\hline $\mathrm{S}_{2}-26^{\text {th }} \mathrm{MW}$ & & $-0.553 *$ & -0.017 & 0.294 & $0.525^{*}$ & -0.068 & 0.166 & -0.328 & -0.152 \\
\hline $\mathrm{S}_{3-}-27^{\text {th }} \mathrm{MW}$ & & -0.318 & -0.143 & 0.212 & 0.312 & -0.157 & -0.123 & -0.080 & 0.070 \\
\hline $\mathrm{S}_{4}-28^{\text {th }} \mathrm{MW}$ & & -0.250 & -0.176 & 0.244 & 0.270 & -0.188 & -0.186 & -0.016 & 0.111 \\
\hline $\mathrm{S}_{1}-25^{\text {th }} \mathrm{MW}$ & \multirow{4}{*}{$\mathrm{V}_{2}-$ RHRG-6083 } & $-0.733^{*}$ & 0.298 & 0.092 & $0.705^{*}$ & 0.070 & 0.602 & $-0.542^{*}$ & -0.482 \\
\hline $\mathrm{S}_{2-} 26^{\text {th }} \mathrm{MW}$ & & $-0.544^{*}$ & -0.020 & 0.286 & $0.517 *$ & -0.070 & 0.149 & -0.313 & -0.134 \\
\hline $\mathrm{S}_{3}-27^{\text {th }} \mathrm{MW}$ & & -0.332 & -0.148 & 0.216 & 0.322 & -0.151 & -0.113 & -0.090 & 0.068 \\
\hline $\mathrm{S}_{4-}-28^{\text {th }} \mathrm{MW}$ & & -0.251 & -0.176 & 0.242 & 0.270 & -0.188 & -0.186 & -0.016 & 0.110 \\
\hline $\mathrm{S}_{1}-25^{\text {th }} \mathrm{MW}$ & \multirow{4}{*}{$\mathrm{V}_{3}-\mathrm{TAG}-24$} & $-0.729 *$ & 0.284 & 0.088 & $0.700 *$ & 0.063 & 0.594 & -0.538 & -0.483 \\
\hline $\mathrm{S}_{2-}-26^{\text {th }} \mathrm{MW}$ & & $-0.548^{*}$ & -0.019 & 0.289 & $0.520 *$ & -0.069 & 0.156 & -0.320 & -0.142 \\
\hline $\mathrm{S}_{3}-27^{\text {th }} \mathrm{MW}$ & & -0.317 & -0.143 & 0.211 & 0.311 & -0.157 & -0.124 & -0.080 & 0.070 \\
\hline $\mathrm{S}_{4-}-28^{\text {th }} \mathrm{MW}$ & & -0.250 & -0.176 & 0.243 & 0.270 & -0.188 & -0.186 & -0.016 & 0.110 \\
\hline $\mathrm{S}_{1}-25^{\text {th }} \mathrm{MW}$ & \multirow{4}{*}{$V_{4-} J L-776$} & $-0.727 *$ & 0.280 & 0.087 & $0.698^{*}$ & 0.061 & $0.592 *$ & $-0.537^{*}$ & -0.483 \\
\hline$S_{2}-26^{\text {th }} M W$ & & $-0.550^{*}$ & -0.018 & 0.291 & $0.522 *$ & -0.069 & 0.159 & -0.322 & -0.145 \\
\hline$S_{3}-27^{\text {th }} M W$ & & -0.330 & -0.147 & 0.216 & 0.320 & -0.152 & -0.114 & -0.088 & 0.068 \\
\hline $\mathrm{S}_{4-}-28^{\text {th }} \mathrm{MW}$ & & -0.245 & -0.178 & 0.251 & 0.268 & -0.187 & -0.191 & -0.011 & 0.115 \\
\hline
\end{tabular}

Tmax- Maximum temperature, Tmin- Minimum temperature, RH-I- Morning humidity, RH-II- Evening humidity, WS- Wind speed, RFRainfall, Epan- Evaporation, BSS- Bright sunshine hours 
Correlation between weather parameter and population of Spodoptera litura larva $\left(\mathrm{mrl}^{-1}\right)$ on groundnut variety $\mathrm{JL}$ 501 at different sowing windows and forewarning models for prediction of population of Spodoptera litura

During first sowing window $25^{\text {th }} \mathrm{MW}\left(\mathrm{S}_{1}\right)$ with variety JL501 the population of Spodoptera litura larva $\left(\mathrm{mrl}^{-1}\right)$ for one week prior $\left(\mathrm{W}^{-1}\right)$ was correlated significantly positive with minimum temperature $(0.216$ and 0.291$)$, evening humidity (0.287 and 0.703) and wind speed (0.458 and 0.598) whereas it was negatively significant with maximum temperature (0.546 and -0.731$)$ and bright sunshine hours (-0.314 and 0.483 ) during kharif season 2017 and 2018, respectively. The overall linear multiple regression analysis was worked out between population of Spodoptera litura on groundnut of W0 week with weather parameters of one week prior $\left(\mathrm{W}^{-1}\right)$ for variety JL-501 and $\mathrm{S}_{1}$ sowing window $\left(25^{\text {th }} \mathrm{MW}\right)$. The results obtained are given as follows. The multiple regression equation is given below:

\section{$\mathrm{Y}=-15.283-0.217(\mathrm{~T} \max )-0.575(\mathrm{~T} \min )+0.329(\mathrm{RH}-$ I)+0.123(RH-II)-0.047(RF)}

An increase of one unit of morning humidity increased the population of Spodoptera litura by 0.32 units and one unit of evening humidity increased the population of Spodoptera litura by 0.12 units. These weather parameter collectively increased the population of Spodoptera litura to an extent of $74 \%\left(\mathrm{R}^{2}=0.74\right)$

During second sowing window $26^{\text {th }} \mathrm{MW}\left(\mathrm{S}_{2}\right)$ with variety JL501 the population of Spodoptera litura larva $\left(\mathrm{mrl}^{-1}\right)$ for one week prior (W-1) was correlated significantly positive with evening humidity $(0.255$ and 0.525$)$ and wind speed $(0.205$ and 0.166$)$ whereas it was negatively significant with maximum temperature (-0.484 and -0.553) and bright sunshine hours (-0.130 and -0.152) during kharif season 2017 and 2018, respectively. The overall linear multiple regression analysis was worked out between population of Spodoptera litura on groundnut of W0 week with weather parameters of one week prior (W-1) for variety JL-501 and $\mathrm{S}_{2}$ sowing window $\left(26^{\text {th }} \mathrm{MW}\right)$. The results obtained are given as follows. The multiple regression equation is given below:

\section{$\mathrm{Y}=-21.043+0.159(\mathrm{Tmax})-1.105(\mathrm{Tmin})+0.349(\mathrm{RH}-$ I) +0.175 (RH-II)-0.038(RF)}

An increase of one unit of maximum temperature increased the population of Spodoptera litura by 0.15 units, one unit of morning humidity increased the population of Spodoptera litura by 0.34 units and one unit of evening humidity increased the population of Spodoptera litura by 0.17 units. These weather parameter collectively increased the population of Spodoptera litura to an extent of $83 \%\left(\mathrm{R}^{2}=0.83\right)$

During third sowing window $27^{\text {th }} \mathrm{MW}\left(\mathrm{S}_{3}\right)$ with variety JL501 the population of Spodoptera litura larva $\left(\mathrm{mrl}^{-1}\right)$ for one week prior (W-1) was correlated significantly positive with evening humidity $(0.232$ and 0.312$)$ whereas it was negatively significant with maximum temperature $(-0.279$ and -0.318$)$ during kharif season 2017 and 2018, respectively. The overall linear multiple regression analysis was worked out between population of Spodoptera litura on groundnut of W0 week with weather parameters of one week prior (W-1) for variety JL-501 and $\mathrm{S}_{3}$ sowing window $\left(27^{\text {th }} \mathrm{MW}\right)$. The results obtained are given as follows. The multiple regression equation is given below:

\section{$\mathrm{Y}=-17.436+0.919(\mathrm{Tmax})-2.071(\mathrm{Tmin})+0.179(\mathrm{RH}-$ I) $+0.325(\mathrm{RH}-\mathrm{II})-0.037(\mathrm{RF})$}

An increase of one unit of maximum temperature increased the population of Spodoptera litura by 0.91 units, one unit of morning humidity increased the population of Spodoptera litura by 0.17 units and one unit of evening humidity increased the population of Spodoptera litura by 0.32 units. These weather parameter collectively increased the population of Spodoptera litura to an extent of $69 \%\left(\mathrm{R}^{2}=0.69\right)$

During fourth sowing window $28^{\text {th }} \mathrm{MW}\left(\mathrm{S}_{4}\right)$ with variety JL501 the population of Spodoptera litura larva $\left(\mathrm{mrl}^{-1}\right)$ for one week prior (W-1) was correlated significantly positive with evening humidity $(0.175$ and 0.270$)$ whereas it was negatively significant with maximum temperature $(-0.185$ and -0.250$)$ during kharif season 2017 and 2018, respectively. The overall linear multiple regression analysis was worked out between population of Spodoptera litura on groundnut of W0 week with weather parameters of one week prior (W-1) for variety JL-501 and $\mathrm{S}_{4}$ sowing window $\left(28^{\text {th }} \mathrm{MW}\right)$. The results obtained are given as follows. The multiple regression equation is given below:

\section{$\mathrm{Y}=-32.194+1.283(\mathrm{Tmax})-2.413(\mathrm{Tmin})+0.251(\mathrm{RH}-$ I) +0.413 (RH-II)-0.502(RF)}

An increase of one unit of maximum temperature increased the population of Spodoptera litura by 1.28 units, one unit of morning humidity increased the population of Spodoptera litura by 0.25 units and one unit of evening humidity increased the population of Spodoptera litura by 0.41 units. These weather parameter collectively increased the population of Spodoptera litura to an extent of $72 \%\left(\mathrm{R}^{2}=0.72\right)$

Correlation between weather parameter and population of Spodoptera litura larva $\left(\mathrm{mrl}^{-1}\right)$ on groundnut variety RHRG-6083 at different sowing windows and forewarning models for prediction of population of Spodoptera litura

During first sowing window $25^{\text {th }} \mathrm{MW}\left(\mathrm{S}_{1}\right)$ with variety RHRG-6083 the population of Spodoptera litura larva $\left(\mathrm{mrl}^{-1}\right)$ for one week prior (W-1) was correlated significantly positive with minimum temperature (0.214 and 0.298$)$, evening humidity (0.290 and 0.705$)$ and wind speed $(0.467$ and 0.602$)$ whereas it was negatively significant with maximum temperature (-0.554 and -0.733) and bright sunshine hours (0.310 and -0.482) during kharif season 2017 and 2018, respectively. The overall linear multiple regression analysis was worked out between population of Spodoptera litura on groundnut of W0 week with weather parameters of one week prior (W-1) for variety RHRG-6083 and $\mathrm{S}_{1}$ sowing window $\left(25^{\text {th }} \mathrm{MW}\right)$. The results obtained are given as follows. The multiple regression equation is given below:

\section{$\mathrm{Y}=-9.588-0.150(\mathrm{Tmax})-0.357(\mathrm{Tmin})+0.210(\mathrm{RH}-$ I) +0.077 (RH-II)-0.030(RF)}

An increase of one unit of morning humidity increased the population of Spodoptera litura by 0.21 units and one unit of evening humidity increased the population of Spodoptera litura by 0.41 units. These weather parameter collectively increased the population of Spodoptera litura to an extent of $73 \%\left(\mathrm{R}^{2}=0.73\right)$

During second sowing window $26^{\text {th }} \mathrm{MW}\left(\mathrm{S}_{2}\right)$ with variety RHRG-6083 the population of Spodoptera litura larva $\left(\mathrm{mrl}^{-1}\right)$ 
for one week prior (W-1) was correlated significantly positive with evening humidity $(0.252$ and 0.517$)$ and wind speed $(0.205$ and 0.149$)$ whereas it was negatively significant with maximum temperature (-0.481 and -0.544) and bright sunshine hours (-0.132 and -0.152) during kharif season 2017 and 2018, respectively. The overall linear multiple regression analysis was worked out between population of Spodoptera litura on groundnut of W0 week with weather parameters of one week prior (W-1) for variety RHRG-6083 and $\mathrm{S}_{2}$ sowing window $\left(26^{\text {th }} \mathrm{MW}\right)$. The results obtained are given as follows. The multiple regression equation is given below:

\section{$\mathrm{Y}=-13.321+0.108(\mathrm{Tmax})-0.727(\mathrm{Tmin})+0.223(\mathrm{RH}-$ \\ I) +0.115 (RH-II)-0.025(RF)}

An increase of one unit of maximum temperature increased the population of Spodoptera litura by 0.10 units, one unit of morning humidity increased the population of Spodoptera litura by 0.22 units and one unit of evening humidity increased the population of Spodoptera litura by 0.11 units. These weather parameter collectively increased the population of Spodoptera litura to an extent of $85 \%\left(\mathrm{R}^{2}=0.85\right)$

During third sowing window $27^{\text {th }} \mathrm{MW}\left(\mathrm{S}_{3}\right)$ with variety RHRG-6083 the population of Spodoptera litura larva $\left(\mathrm{mrl}^{-1}\right)$ for one week prior (W-1) was correlated significantly positive with evening humidity $(0.234$ and 0.322$)$ whereas it was negatively significant with maximum temperature $(-0.288$ and -0.332) during kharif season 2017 and 2018, respectively. The overall linear multiple regression analysis was worked out between population of Spodoptera litura on groundnut of W0 week with weather parameters of one week prior $(\mathrm{W}-1)$ for variety RHRG-6083 and $\mathrm{S}_{3}$ sowing window $\left(27^{\text {th }} \mathrm{MW}\right)$. The results obtained are given as follows. The multiple regression equation is given below:

\section{$\mathrm{Y}=-10.714+0.574(\mathrm{Tmax})-1.334(\mathrm{Tmin})+0.117(\mathrm{RH}-$ I)+0.207(RH-II)-0.023(RF)}

An increase of one unit of maximum temperature increased the population of Spodoptera litura by 0.57 units, one unit of morning humidity increased the population of Spodoptera litura by 0.11 units and one unit of evening humidity increased the population of Spodoptera litura by 0.20 units. These weather parameter collectively increased the population of Spodoptera litura to an extent of $66 \%\left(\mathrm{R}^{2}=0.66\right)$

During fourth sowing window $28^{\text {th }} \mathrm{MW}\left(\mathrm{S}_{4}\right)$ with variety RHRG-6083 the population of Spodoptera litura larva $\left(\mathrm{mrl}^{-1}\right)$ for one week prior (W-1) was correlated significantly positive with evening humidity $(0.177$ and 0.270$)$ whereas it was negatively significant with maximum temperature $(-0.187$ and -0.251) during kharif season 2017 and 2018, respectively. The overall linear multiple regression analysis was worked out between population of Spodoptera litura on groundnut of W0 week with weather parameters of one week prior (W-1) for variety RHRG-6083 and $\mathrm{S}_{4}$ sowing window $\left(28^{\text {th }} \mathrm{MW}\right)$. The results obtained are given as follows. The multiple regression equation is given below:

\section{$\mathrm{Y}=-20.737+0.827(\mathrm{Tmax})-1.558(\mathrm{Tmin})+0.162(\mathrm{RH}-$ I) +0.267 (RH-II)-0.037(RF)}

An increase of one unit of maximum temperature increased the population of Spodoptera litura by 0.85 units, one unit of morning humidity increased the population of Spodoptera litura by 0.16 units and one unit of evening humidity increased the population of Spodoptera litura by 0.26 units. These weather parameter collectively increased the population of Spodoptera litura to an extent of $75 \%\left(\mathrm{R}^{2}=0.75\right)$

Correlation between weather parameter and population of Spodoptera litura larva $\left(\mathrm{mrl}^{-1}\right)$ on groundnut variety TAG24 at different sowing windows and forewarning models for prediction of population of Spodoptera litura

During first sowing window $25^{\text {th }} \mathrm{MW}\left(\mathrm{S}_{1}\right)$ with variety TAG24 the population of Spodoptera litura larva $\left(\mathrm{mrl}^{-1}\right)$ for one week prior (W-1) was correlated significantly positive with evening humidity (0.289 and 0.700$)$, minimum temperature $(0.216$ and 0.284$)$ and wind speed (0.464 and 0.594) whereas it was negatively significant with maximum temperature (0.550 and -0.729$)$ and bright sunshine hours (-0.313 and 0.483 ) during kharif season 2017 and 2018, respectively. The overall linear multiple regression analysis was worked out between population of Spodoptera litura on groundnut of W0 week with weather parameters of one week prior (W-1) for variety TAG-24 and $\mathrm{S}_{1}$ sowing window $\left(25^{\text {th }} \mathrm{MW}\right)$. The results obtained are given as follows. The multiple regression equation is given below:

\section{$\mathrm{Y}=-16.046-0.227(\mathrm{Tmax})-0.634(\mathrm{Tmin})+0.349(\mathrm{RH}-$ I) $+0.135(\mathrm{RH}-\mathrm{II})-0.051(\mathrm{RF})$}

An increase of one unit of morning humidity increased the population of Spodoptera litura by 0.34 units and one unit of evening humidity increased the population of Spodoptera litura by 0.13 units. These weather parameter collectively increased the population of Spodoptera litura to an extent of $71 \%\left(\mathrm{R}^{2}=0.71\right)$

During second sowing window $26^{\text {th }} \mathrm{MW}\left(\mathrm{S}_{2}\right)$ with variety TAG-24 the population of Spodoptera litura larva $\left(\mathrm{mrl}^{-1}\right)$ for one week prior (W-1) was correlated significantly positive with evening humidity $(0.257$ and 0.520$)$ and wind speed (0.206 and 0.156$)$ whereas it was negatively significant with maximum temperature (-0.486 and -0.548) during kharif season 2017 and 2018, respectively. The overall linear multiple regression analysis was worked out between population of Spodoptera litura on groundnut of W0 week with weather parameters of one week prior (W-1) for variety TAG-24 and $\mathrm{S}_{2}$ sowing window $\left(26^{\text {th }} \mathrm{MW}\right)$. The results obtained are given as follows. The multiple regression equation is given below:

\section{$\mathrm{Y}=-22.464+0.177(\mathrm{Tmax})-1.214(\mathrm{Tmin})+0.376(\mathrm{RH}-$ I)+0.192(RH-II)-0.042(RF)}

An increase of one unit of maximum temperature increased the population of Spodoptera litura by 0.17 units, one unit of morning humidity increased the population of Spodoptera litura by 0.37 units and one unit of evening humidity increased the population of Spodoptera litura by 0.19 units. These weather parameter collectively increased the population of Spodoptera litura to an extent of $87 \%\left(\mathrm{R}^{2}=0.87\right)$

During third sowing window $27^{\text {th }} \mathrm{MW}\left(\mathrm{S}_{3}\right)$ with variety TAG24 the population of Spodoptera litura larva $\left(\mathrm{mrl}^{-1}\right)$ for one week prior (W-1) was correlated significantly positive with evening humidity $(0.234$ and 0.311$)$ whereas it was negatively significant with maximum temperature $(-0.289$ and -0.317$)$ during kharif season 2017 and 2018, respectively. The overall linear multiple regression analysis was worked out between 
population of Spodoptera litura on groundnut of W0 week with weather parameters of one week prior (W-1) for variety TAG-24 and $\mathrm{S}_{3}$ sowing window $\left(27^{\text {th }} \mathrm{MW}\right)$. The results obtained are given as follows. The multiple regression equation is given below:

\section{$\mathrm{Y}=-18.546+0.986(\mathrm{Tmax})-2.245(\mathrm{Tmin})+0.196(\mathrm{RH}-$ I)+0.351(RH-II)-0.039(RF)}

An increase of one unit of maximum temperature increased the population of Spodoptera litura by 0.98 units, one unit of morning humidity increased the population of Spodoptera litura by 0.19 units and one unit of evening humidity increased the population of Spodoptera litura by 0.35 units. These weather parameter collectively increased the population of Spodoptera litura to an extent of $73 \%\left(\mathrm{R}^{2}=0.73\right)$

During fourth sowing window $28^{\text {th }} \mathrm{MW}\left(\mathrm{S}_{4}\right)$ with variety TAG-24 the population of Spodoptera litura larva $\left(\mathrm{mrl}^{-1}\right)$ for one week prior (W-1) was correlated significantly positive with evening humidity $(0.175$ and 0.200$)$ whereas it was negatively significant with maximum temperature $(-0.186$ and -0.250) during kharif season 2017 and 2018, respectively. The overall linear multiple regression analysis was worked out between population of Spodoptera litura on groundnut of W0 week with weather parameters of one week prior $(\mathrm{W}-1)$ for variety TAG-24 and $\mathrm{S}_{4}$ sowing window $\left(28^{\text {th }} \mathrm{MW}\right)$. The results obtained are given as follows. The multiple regression equation is given below:

\section{$\mathrm{Y}=-34.908+1.391(\mathrm{Tmax})-2.617(\mathrm{Tmin})+0.273(\mathrm{RH}-$ I) +0.448 (RH-II)-0.056(RF)}

An increase of one unit of maximum temperature increased the population of Spodoptera litura by 1.39 units, one unit of morning humidity increased the population of Spodoptera litura by 0.27 units and one unit of evening humidity increased the population of Spodoptera litura by 0.44 units. These weather parameter collectively increased the population of Spodoptera litura to an extent of $71 \%\left(\mathrm{R}^{2}=0.71\right)$

Correlation between weather parameter and population of Spodoptera litura larva $\left(\mathrm{mrl}^{-1}\right)$ on groundnut variety $\mathrm{JL}$ 776 at different sowing windows and forewarning models for prediction of population of Spodoptera litura

During first sowing window $25^{\text {th }} \mathrm{MW}\left(\mathrm{S}_{1}\right)$ with variety JL776 the population of Spodoptera litura larva $\left(\mathrm{mrl}^{-1}\right)$ for one week prior (W-1) was correlated significantly positive with minimum temperature $(0.220$ and 0.280$)$, evening humidity (0.288 and 0.698) and wind speed (0.459 and 0.592) whereas it was negatively significant with maximum temperature (0.545 and -0.727$)$ and bright sunshine hours (-0.316 and 0.483) during kharif season 2017 and 2018, respectively. The overall linear multiple regression analysis was worked out between population of Spodoptera litura on groundnut of W0 week with weather parameters of one week prior $(\mathrm{W}-1)$ for variety JL-776 and $\mathrm{S}_{1}$ sowing window $\left(25^{\text {th }} \mathrm{MW}\right)$. The results obtained are given as follows. The multiple regression equation is given below:

\section{$\mathrm{Y}=-6.680-0.087(\mathrm{Tmax})-0.271(\mathrm{Tmin})-0.144(\mathrm{RH}-$ I) +0.057 (RH-II)-0.021(RF)}

An increase of one unit of evening humidity increased the population of Spodoptera litura by 0.57 units to an extent of
$72 \%\left(\mathrm{R}^{2}=0.72\right)$

During second sowing window $26^{\text {th }} \mathrm{MW}\left(\mathrm{S}_{2}\right)$ with variety JL776 the population of Spodoptera litura larva $\left(\mathrm{mrl}^{-1}\right)$ for one week prior (W-1) was correlated significantly positive with evening humidity $(0.241$ and 0.522$)$ and wind speed $(0.205$ and 0.159$)$ whereas it was negatively significant with maximum temperature $(-0.470$ and -0.550$)$ and bright sunshine hours (-0.142 and -0.185) during kharif season 2017 and 2018, respectively. The overall linear multiple regression analysis was worked out between population of Spodoptera litura on groundnut of W0 week with weather parameters of one week prior (W-1) for variety JL-776 and $\mathrm{S}_{2}$ sowing window $\left(26^{\text {th }} \mathrm{MW}\right)$. The results obtained are given as follows. The multiple regression equation is given below:

\section{$\mathrm{Y}=-9.496+0.069(\mathrm{Tmax})-0.491(\mathrm{Tmin})+0.157(\mathrm{RH}-$ I) $+0.078(\mathrm{RH}-\mathrm{II})-0.017(\mathrm{RF})$}

An increase of one unit of maximum temperature increased the population of Spodoptera litura by 0.06 units, one unit of morning humidity increased the population of Spodoptera litura by 0.15 units and one unit of evening humidity increased the population of Spodoptera litura by 0.07 units. These weather parameter collectively increased the population of Spodoptera litura to an extent of $84 \%\left(\mathrm{R}^{2}=0.84\right)$

During third sowing window $27^{\text {th }} \mathrm{MW}\left(\mathrm{S}_{3}\right)$ with variety JL776 the population of Spodoptera litura larva $\left(\mathrm{mrl}^{-1}\right)$ for one week prior (W-1) was correlated significantly positive with evening humidity $(0.232$ and 0.320$)$ whereas it was negatively significant with maximum temperature $(-0.280$ and -0.330$)$ during kharif season 2017 and 2018, respectively. The overall linear multiple regression analysis was worked out between population of Spodoptera litura on groundnut of W0 week with weather parameters of one week prior $(\mathrm{W}-1)$ for variety JL-776 and $\mathrm{S}_{3}$ sowing window $\left(27^{\text {th }} \mathrm{MW}\right)$. The results obtained are given as follows. The multiple regression equation is given below:

\section{$\mathrm{Y}=-7.725+0.405(\mathrm{Tmax})-0.926(\mathrm{Tmin})+0.081(\mathrm{RH}-$ I) $+0.145(\mathrm{RH}-\mathrm{II})-0.016(\mathrm{RF})$}

An increase of one unit of maximum temperature increased the population of Spodoptera litura by 0.40 units, one unit of morning humidity increased the population of Spodoptera litura by 0.08 units and one unit of evening humidity increased the population of Spodoptera litura by 0.14 units. These weather parameter collectively increased the population of Spodoptera litura to an extent of $69 \%\left(\mathrm{R}^{2}=0.69\right)$

During fourth sowing window $28^{\text {th }} \mathrm{MW}\left(\mathrm{S}_{4}\right)$ with variety JL776 the population of Spodoptera litura larva $\left(\mathrm{mrl}^{-1}\right)$ for one week prior (W-1) was correlated significantly positive with evening humidity $(0.157$ and 0.268$)$ whereas it was negatively significant with maximum temperature $(-0.159$ and -0.245$)$ during kharif season 2017 and 2018, respectively. The overall linear multiple regression analysis was worked out between population of Spodoptera litura on groundnut of W0 week with weather parameters of one week prior (W-1) for variety JL-776 and $\mathrm{S}_{4}$ sowing window $\left(28^{\text {th }} \mathrm{MW}\right)$. The results obtained are given as follows. The multiple regression equation is given below:

$Y=-15.596+0.590(\mathrm{Tmax})-1.076(\mathrm{Tmin})+0.118(\mathrm{RH}-$
$\mathrm{I})+0.187(\mathrm{RH}-\mathrm{II})-0.024(\mathrm{RF})$ 
An increase of one unit of maximum temperature increased the population of Spodoptera litura by 0.59 units, one unit of morning humidity increased the population of Spodoptera litura by 0.11 units and one unit of evening humidity increased the population of Spodoptera litura by 0.18 units.
These weather parameter collectively increased the population of Spodoptera litura to an extent of $77 \%\left(\mathrm{R}^{2}=0.77\right)$

Similar results were reported by Sojitra (1990) ${ }^{[15]}$ and Yadav et al. $(2015)^{[16]}$.

Table 8: Forewarning model developed for one week prior (W-1) prediction of incidence of Spodoptera litura larva per meter row length (mrl ${ }^{-1}$ ) on groundnut

\begin{tabular}{|c|c|c|c|}
\hline \multicolumn{2}{|c|}{ Treatment } & \multirow{2}{*}{ Forewarning model's } & \multirow{2}{*}{$\mathbf{r}^{2}$ Value } \\
\hline Sowing window & Variety & & \\
\hline $\mathrm{S}_{1}-25^{\text {th }} \mathrm{MW}$ & \multirow{4}{*}{$\mathrm{V}_{1}-\mathrm{JL}-501$} & $\mathrm{Y}=-15.283-0.217(\mathrm{Tmax})-0.575(\mathrm{Tmin})+0.329(\mathrm{RH}-\mathrm{I})+0.123(\mathrm{RH}-\mathrm{II})-0.047(\mathrm{RF})$ & 0.74 \\
\hline $\mathrm{S}_{2}-26^{\text {th }} \mathrm{MW}$ & & $\mathrm{Y}=-21.043+0.159(\mathrm{Tmax})-1.105(\mathrm{Tmin})+0.349(\mathrm{RH}-\mathrm{I})+0.175(\mathrm{RH}-\mathrm{II})-0.038(\mathrm{RF})$ & 0.83 \\
\hline $\mathrm{S}_{3}-27^{\text {th }} \mathrm{MW}$ & & $\mathrm{Y}=-17.436+0.919(\mathrm{Tmax})-2.071(\mathrm{Tmin})+0.179(\mathrm{RH}-\mathrm{I})+0.325(\mathrm{RH}-\mathrm{II})-0.037(\mathrm{RF})$ & 0.69 \\
\hline $\mathrm{S}_{4}-28^{\text {th }} \mathrm{MW}$ & & $\mathrm{Y}=-32.194+1.283(\mathrm{Tmax})-2.413(\mathrm{Tmin})+0.251(\mathrm{RH}-\mathrm{I})+0.413(\mathrm{RH}-\mathrm{II})-0.502(\mathrm{RF})$ & 0.72 \\
\hline $\mathrm{S}_{1}-25^{\text {th }} \mathrm{MW}$ & \multirow{4}{*}{ V $2-$ RHRG-6083 } & $\mathrm{Y}=-9.588-0.150(\mathrm{Tmax})-0.357(\mathrm{Tmin})+0.210(\mathrm{RH}-\mathrm{I})+0.077(\mathrm{RH}-\mathrm{II})-0.030(\mathrm{RF})$ & 0.73 \\
\hline $\mathrm{S}_{2}-26^{\text {th }} \mathrm{MW}$ & & $\mathrm{Y}=-13.321+0.108(\mathrm{Tmax})-0.727(\mathrm{Tmin})+0.223(\mathrm{RH}-\mathrm{I})+0.115(\mathrm{RH}-\mathrm{II})-0.025(\mathrm{RF})$ & 0.85 \\
\hline $\mathrm{S}_{3}-27^{\text {th }} \mathrm{MW}$ & & $\mathrm{Y}=-10.714+0.574(\mathrm{Tmax})-1.334(\mathrm{Tmin})+0.117(\mathrm{RH}-\mathrm{I})+0.207(\mathrm{RH}-\mathrm{II})-0.023(\mathrm{RF})$ & 0.66 \\
\hline $\mathrm{S}_{4}-28^{\text {th }} \mathrm{MW}$ & & $\mathrm{Y}=-20.737+0.827(\mathrm{Tmax})-1.558(\mathrm{Tmin})+0.162(\mathrm{RH}-\mathrm{I})+0.267(\mathrm{RH}-\mathrm{II})-0.037(\mathrm{RF})$ & 0.75 \\
\hline $\mathrm{S}_{1}-25^{\text {th }} \mathrm{MW}$ & \multirow{4}{*}{$\mathrm{V}_{3}-\mathrm{TAG}-24$} & $\mathrm{Y}=-16.046-0.227(\mathrm{Tmax})-0.634(\mathrm{Tmin})+0.349(\mathrm{RH}-\mathrm{I})+0.135(\mathrm{RH}-\mathrm{II})-0.051(\mathrm{RF})$ & 0.71 \\
\hline $\mathrm{S}_{2}-26^{\text {th }} \mathrm{MW}$ & & $\mathrm{Y}=-22.464+0.177(\mathrm{Tmax})-1.214(\mathrm{Tmin})+0.376(\mathrm{RH}-\mathrm{I})+0.192(\mathrm{RH}-\mathrm{II})-0.042(\mathrm{RF})$ & 0.87 \\
\hline $\mathrm{S}_{3}-27^{\text {th }} \mathrm{MW}$ & & $\mathrm{Y}=-18.546+0.986(\mathrm{Tmax})-2.245(\mathrm{Tmin})+0.196(\mathrm{RH}-\mathrm{I})+0.351(\mathrm{RH}-\mathrm{II})-0.039(\mathrm{RF})$ & 0.73 \\
\hline $\mathrm{S}_{4}-28^{\text {th }} \mathrm{MW}$ & & $\mathrm{Y}=-34.908+1.391(\mathrm{Tmax})-2.617(\mathrm{Tmin})+0.273(\mathrm{RH}-\mathrm{I})+0.448(\mathrm{RH}-\mathrm{II})-0.056(\mathrm{RF})$ & 0.71 \\
\hline $\mathrm{S}_{1}-25^{\text {th }} \mathrm{MW}$ & \multirow{4}{*}{$\mathrm{V}_{4}-\mathrm{JL}-776$} & $\mathrm{Y}=-6.680-0.087(\mathrm{Tmax})-0.271(\mathrm{Tmin})-0.144(\mathrm{RH}-\mathrm{I})+0.057(\mathrm{RH}-\mathrm{II})-0.021(\mathrm{RF})$ & 0.72 \\
\hline $\mathrm{S}_{2}-26^{\text {th }} \mathrm{MW}$ & & $\mathrm{Y}=-9.496+0.069(\mathrm{Tmax})-0.491(\mathrm{Tmin})+0.157(\mathrm{RH}-\mathrm{I})+0.078(\mathrm{RH}-\mathrm{II})-0.017(\mathrm{RF})$ & 0.84 \\
\hline $\mathrm{S}_{3}-27^{\text {th }} \mathrm{MW}$ & & $\mathrm{Y}=-7.725+0.405(\mathrm{Tmax})-0.926(\mathrm{Tmin})+0.081(\mathrm{RH}-\mathrm{I})+0.145(\mathrm{RH}-\mathrm{II})-0.016(\mathrm{RF})$ & 0.69 \\
\hline $\mathrm{S}_{4}-28^{\text {th }} \mathrm{MW}$ & & $\mathrm{Y}=-15.596+0.590(\mathrm{Tmax})-1.076(\mathrm{Tmin})+0.118(\mathrm{RH}-\mathrm{I})+0.187(\mathrm{RH}-\mathrm{II})-0.024(\mathrm{RF})$ & 0.77 \\
\hline
\end{tabular}

Tmax- Maximum temperature, Tmin- Minimum temperature, RH-I- Morning humidity, RH-II- Evening humidity, WS- Wind speed, RFRainfall, Epan- Evaporation, BSS- Bright sunshine hours

\section{Conclusion}

The correlation of weather parameters with incidence of Spodoptera litura showed that the population of Spodoptera litura was found to have significant and positive correlation with evening relative humidity whereas maximum temperature and rainfall showed negative correlation with seasonal incidence of Spodoptera litura. Prediction of Spodoptera litura populations in different sowing window based on regression equations $\left(\mathrm{R}^{2}\right) 72$ to 83 per cent validation based on different weather parameters for variety JL-501, $\left(\mathrm{R}^{2}\right) 66$ to 85 per cent validation based on different weather parameter for variety RHRG-6083, $\left(\mathrm{R}^{2}\right) 71$ to 87 per cent validation based on different weather parameter for variety TAG-24 and $\left(\mathrm{R}^{2}\right) 69$ to 84 per cent validation based on different weather parameter for variety JL-776 for the prediction of Spodoptera litura population.

\section{References}

1. Wightman JA, Amin PW. Groundnut pests and their control in the semi-arid tropics. Tropical Pest Management. 2008; 34:218-226.

2. Murthy S, Thippaiah M, Kitturmmath MS. Effect of neem formulations on larvae of tobacco cutworm, Spodoptera litura (Fab.). Insect Environment. 2006; 12:84-85.

3. Dhir BC, Mohapatra HK, Senapati B. Assessment of crop loss in groundnut due to tobacco caterpillar, Spodoptera litura (F.). Indian J Plant Protect. 1992; 20:215-217.

4. Isman MB, Machial CM, Miresmailli S, Bainard LD. Essential oil based pesticides: new insights from old chemistry. In: Pesticide chemistry, H. Ohkawa and $\mathrm{H}$ Miyagawa, (Eds): Wiley. Weinheim, 2007, 113.

5. Saheb YP, Hari Prasad KV, Swarajya Lakshmi K, Sailaja Rani J. Mechanisms of resistance in groundnut genotypes against leaf bud borer, Anarsia ephippias (Meyrick). Journal of Entomology and Zoology Studies. 2018; 6(2):694-705.

6. Raghunathan V. Integrated Pest Management: Eco- friendly approach. The Hindu Survey of Indian Agriculture, 1996, 155.

7. Singh SP. Pest Management. The Eco-friendly approach. The Hindu Survey of Indian Agriculture, 1999, 175-184.

8. Snedecor GW, Cochran WG. Statistical methods. $6^{\text {th }}$ edition, Lowa State University Press, USA, 1968.

9. Draper NR, Smith H. Applied Regression Analysis, edi. Wiley, 1998, 3.

10. Rathod RR. Population dynamics, efficacy and economics of newer insecticides against groundnut (Arachis hypogaea L.) pest complex. M. Sc. Thesis, Junagadh Agricultural University, Junagadh, 2006 1-3.

11. Arvind Kumar. Influence of weather parameters on pheromone traps catches of Spodoptera litura Febr. J Agri. 2014; (4):238-241.

12. Harish G, Nataraja MV, Jasrotia P, Holajjer P, Savaliya $\mathrm{SD}$, Gajera M. Impact of weather on the occurrence pattern of insect pests on groundnut. Legume Research. 2014; 38(4):524-535.

13. Roopa M, Ashok kumar CT. Seasonal incidence of pests of capsicum in Bangalore conditions of Karnataka, India. Global Journal of Biology, Agriculture \& Health Sciences. 2014; 3(3):203-207.

14. Nath P, Rai SN, Keval R. Effect of sowing dates on the tobacco caterpillar [Spodoptera litura (Fabricius)], population, leaf damage and yield of groundnut. International Journal of Research- Granthaalayah. 2017; ISSN- 2350- 0530 (O), ISSN- 2394-3629(P).

15. Sojitra BP. Population dynamics of major soybean pests along with bionomics of grey semilooper, Amynaocto (Guenee) on different hosts and its chemical control. M.Sc. (Agri.) Thesis submitted to the Gujarat Agricultural University, Sardar Krushinagar, 1990.

16. Yadav S, Agnihotri M, Bisht RS. Seasonal incidence of insect-pests of blackgram, Vigna mungo (Linn.) and its correlation with abiotic factors. Agric. Sci. Digest. 2015; 35(2):146-148. 